\title{
碳纳米管添加剂对质子交换膜燃料电池低铂载量膜电极 性能的影响研究
}

\author{
崔丽瑞张劲 孙一炎卢善富* 相艳* \\ (北京航空航天大学空间与环境学院 仿生能源材料与器件北京市重点实验室 北京 100191)
}

\begin{abstract}
摘要 以碳纳米管 $(\mathrm{CNT})$ 作为低铂载量膜电极 $(\mathrm{CCM})$ 催化层 $\left(0.1 \mathrm{mg}_{\mathrm{P}}{ }^{\bullet} \mathrm{cm}^{-2}\right)$ 添加剂, 研究了 $\mathrm{CNT}$ 的添加方式对催化层微 观结构以及膜电极性能的影响. 结果表明, 与常规低铂载量催化层相比, 在其表面喷涂一层 CNT 或将 CNT 均匀分散到 $\mathrm{Pt} / \mathrm{C}$ 催化层中均有利于提升低铂载量膜电极的输出性能, 在 $70{ }^{\circ} \mathrm{C}$ 和 $100 \%$ 相对湿度条件下最高输出功率比常规低铂载 量膜电极的 $0.522 \mathrm{~W} \cdot \mathrm{cm}^{-2}$ 分别提升了 $22.4 \%$ 和 $60.0 \%$, 并且均匀分散添加方式优于分层添加方式. 其原因在于分层添加 CNT 后改善了低铂催化层和气体扩散层之间的接触界面，降低了催化层与扩散层间的接触电阻，而均匀分散添加方式 除了可降低界面接触电阻外, 还显著改善了低铂催化层中的气体传输, 大幅提升了 $\mathrm{Pt}$ 催化剂的利用效率, 使得膜电极 电化学反应电阻明显降低. 进一步对均匀分散添加方式中 CNT 的载量进行优化, 表明 CNT 添加量为 $37.5 \mu \mathrm{g} \cdot \mathrm{cm}^{-2}$ 时电 池输出性能最佳, $70{ }^{\circ} \mathrm{C}$ 和 $100 \%$ 相对湿度条件下的最大输出功率达到 $0.91 \mathrm{~W} \cdot \mathrm{cm}^{-2}$. 本研究工作表明, 将 CNT 均匀分散 添加到催化层中是一种有效提升低铂载量膜电极性能的方法.
\end{abstract}

关键词＼cjkstart质子交换膜燃料电池; 催化层; 碳纳米管; 低铂; 膜电极

\section{Effect of Addition of Carbon Nanotubes on the Performance of a Low Pt Loading Membrane-Electrode-Assembly in Proton Exchange Membrane Fuel Cells}

\author{
Cui, Lirui $\quad$ Zhang, Jin Sun, Yiyan Lu, Shanfu* Xiang, Yan* \\ (Beijing Key Laboratory of Bio-inspired Energy Materials and Devices, School of Space and Environment, \\ Beihang University, Beijing 100191)
}

\begin{abstract}
The cell performance and Pt utilization of low-Pt proton exchange membrane fuel cells (PEMFCs) have been significantly improved through incorporating carbon materials into the conventional $\mathrm{Pt} / \mathrm{C}$ catalytic layer of the membrane electrode assembly (MEA). However, the introduction methods for the carbon materials have not been investigated. In this work, carbon nanotube (CNT) as an additive was added to the low-Pt loading catalytic layer $\left(0.1 \mathrm{mg}_{\mathrm{Pt}} \bullet \mathrm{cm}^{-2}\right)$ by two methods: a separated CNT layer deposited on the top of the conventional Pt/C layer (CCM-1) and a mixture layer by blending CNT and $\mathrm{Pt} / \mathrm{C}$ catalyst (CCM-2). The conventional low-Pt loading catalytic layer was employed as control group (CCM-0). The microstructure of the catalytic layers was characterized by scanning electron microscopy, transmission electron microscopy and nitrogen sorption isotherms method. The electrochemical properties of the catalytic layer and membrane electrode were evaluated by cyclic voltammetry (CV), electrochemical impedance (EIS) and linear scanning voltammetry. The results indicated that the cell performance of the conventional low-Pt loading catalyst coated membrane was improved by the introduction of CNTs in both CCM-1 and CCM-2. Compared to the conventional CCM (CCM-0) with a peak power density of $0.522 \mathrm{~W}$ $\mathrm{cm}^{-2}$ at $70{ }^{\circ} \mathrm{C}$ and $100 \%$ relative humidity (RH) without backpressure, the maximum power densities of CCM-1 and CCM-2 have been improved by $22.4 \%$ and $60.0 \%$ under the same test conditions, respectively. The increased performance of CCM- 1 is believed to result from the enhancement of contact interface between the catalytic layer and the gas diffusion layer in CCM-1 and consequent decrease of the contact resistance. Furthermore, the outstanding power density of CCM- 2 is not only owing to the decreased interface contact resistance between the CCM and the gas diffusion layer, but also due to the significant improvement of gas transmission in the catalytic layer, which leads to the decrease of electrochemical reactant resistance and then improvement of the Pt utilization. That has been confirmed by the Pt utilization of $34.4 \%, 35.6 \%$ and $44.7 \%$ for CCM-0, CCM-1 and CCM-2. In addition, it also was confirmed by the extremely low power output $\left(2.9 \mathrm{~mW} \cdot \mathrm{cm}^{-2}\right)$ of a CCM with only CNT in the catalytic layer when the fuel cell was tested at $70{ }^{\circ} \mathrm{C}$ and $100 \%$ RH without back pressure. In addition, the optimum loading of CNT in the mixed catalytic layer is $37.5 \mu \mathrm{g} \cdot \mathrm{cm}^{-2}$ with the peak power density of 0.91
\end{abstract}

*E-mail: lusf@buaa.edu.cn; xiangy@buaa.edu.cn

Received August 21, 2018; published November 30, 2018.

Supporting information for this article is available free of charge via the Internet at http://sioc-journal.cn.

Project supported by the Key Research and Development Program of Beijing (No. Z171100000917011), the National Natural Science Foundation of China (Nos. 21722601, 21576007) and the Fundamental Research Funds for the Central Universities.

项目受北京市重点研发计划(No. Z171100000917011)、国家自然科学基金(Nos. 21722601, 21576007)和中央高校基本科研业务费专项资助. 
$\mathrm{W} \cdot \mathrm{cm}^{-2}$. This work shows that mixing of CNT and Pt/C catalyst into a catalytic layer is an effective method for improving the Pt utilization and reducing the loading of Pt catalyst.

Keywords proton exchange membrane fuel cells (PEMFCs); catalytic layer; carbon nanotube; low-Pt loading; membrane electrode assembly

\section{1 引言}

化石能源的快速消耗导致环境污染和能源危机日 益加剧, 使得全世界都在积极寻找开发安全和可再生的 清洁能源 ${ }^{[1-5]}$. 质子交换膜燃料电池 (PEMFCs) 由于具有 高效率、高功率密度、清洁无污染、可低温运行以及快 速启动等优点受到世界各国的普遍关注 ${ }^{[6 ~ 8]}$. 质子交换 膜燃料电池的核心部件为膜电极(Membrane Electrode Assembly, MEA), 包括气体扩散层、催化层和质子交换 膜. 催化层是电化学反应发生的场所, 其中质子、电子 和气体在催化剂的表面结合形成水 ${ }^{[9,10]}$. 目前 PEMFCs 采用的催化剂主要为铂 $(\mathrm{Pt})$ 基催化剂. 虽然近年来非铂 催化剂研究取得许多重要进展 ${ }^{[1 \sim 15]}$, 但其催化活性和 稳定性与 Pt 基催化剂仍然存在较大差距 ${ }^{[12]}$, 在短时间 内还难以取代铂基催化剂 ${ }^{[13]}$. Pt 催化剂的资源稀缺及其 高的成本严重制约了 PEMFCs 的产业化进程 ${ }^{[8,16,17]}$. 因 此, 提高 Pt 催化剂的利用率以及降低 Pt 催化剂的用量 是现阶段 PEMFCs 发展的当务之急 ${ }^{[17]}$.

膜电极中催化层的微观结构和电化学性能主要受 催化剂墨水的成分及催化层的制备方法等因素影 响 ${ }^{[18 \sim 20]}$. 其中膜电极的制备方式直接影响着催化层的 微观结构和 Pt 催化剂的利用效率 ${ }^{[18]}$, 因此一系列新的 膜电极制备方法或新的膜电极结构被发展出来以提高 $\mathrm{Pt}$ 催化剂的利用率或降低膜电极中铂催化剂的载量 ${ }^{[21]}$. 采用超声喷涂方法将催化剂墨水直接在电解质膜上制 备薄层亲水催化层 $(\mathrm{CCM})$ 是一种简单有效的降低催化 剂用量的方法, 但该方法制备的膜电极在大电流密度条 件下工作时由于排水能力不足易出现浓差极化; 此外, 超声喷涂方法对降低 Pt 催化剂载量也存在一定的限度.

现有研究表明, 在薄层亲水催化层中引入适量的纳 米碳材料可以提高催化层的孔隙率和孔结构的均匀性, 改善催化层的三相传输界面, 提升催化剂利用效率 ${ }^{[7]}$, 进而提高电池性能 ${ }^{[22 ~ 24]}$. 例如, 廖世军等 ${ }^{[23]}$ 将氮掺杂 的碳纳米管引入到阴极催化层, 在 $70{ }^{\circ} \mathrm{C}$ 和 $100 \%$ 加湿 的条件下, 总铂载量为 $0.3 \mathrm{mg}_{\mathrm{Pt}} \bullet \mathrm{cm}^{-2}$ 的单电池最大输出 功率从 $0.781 \mathrm{~W} \cdot \mathrm{cm}^{-2}$ 提高到 $0.997 \mathrm{~W} \cdot \mathrm{cm}^{-2}$.

本工作拟采用超声喷涂方法探讨将碳纳米管(CNT) 作为添加剂添加至在低铂载量 $\left(0.1 \mathrm{mg}_{\mathrm{P}} \bullet^{\bullet} \mathrm{cm}^{-2}\right)$ 阴极催化 层中, 考察 CNT 的添加方式对催化层微观结构以及膜 电极性能的影响. 本研究中 CNT 的添加方式分为以下 两种: 一种是先将碳载铂 $(\mathrm{Pt} / \mathrm{C})$ 催化剂墨水喷涂到 Nafion 211 膜上得到催化剂, 然后再将一定量的 CNT 喷 涂到催化剂层上形成覆盖层, 得到 CCM-1, 目的是改善 催化层与气体扩散层的界面接触, 降低接触电阻; 另外
一种是将 $\mathrm{Pt} / \mathrm{C}$ 催化剂和 $\mathrm{CNT}$ 均匀混合配制墨水, 然后 采用超声喷涂在 Nafion 211 膜上, 得到 CCM-2. 此外, 制备未添加 CNT 的膜电极(CCM-0) 以及只采用 CNT 作 为阴极催化层的膜电极 (CCM-3) 作为对照样品进行对 比. 采用扫描电子显微镜(SEM)、透射电子显微镜(TEM) 和麦克 ASAP 2460 物理吸附仪对催化层微观形貌和结 构进行表征; 采用循环伏安法、交流阻抗法、线性伏安 法等方法对催化层电化学行为和膜电极的电化学性能 进行了评估. 研究结果表明两种 CNT 添加方式均有利 于提升低铂膜电极的输出性能, 且 CNT 均匀分散于催 化层中更有利于低铂膜电极性能的提升.

\section{2 结果与讨论}

\subsection{CNT 添加方式对催化层微观结构的影响}

本研究中所制备的三种不同 CCMs 阴极催化层的 微观结构如图 1 所示. 图 $1 \mathrm{a}$ 和 $1 \mathrm{~d}$ 为常规低铂载量膜电 极 $(\mathrm{CCM}-0)$ 阴极催化层断面的不同放大倍数 SEM 图像. 从图中可以看到 $\mathrm{CCM}-0$ 催化层的平均厚度约为 $1.0 \mu \mathrm{m}$, 其结构较为均匀, 但孔隙率不高, 且大部分孔径在 100 $\mathrm{nm}$ 以下. 在该催化层结构基础上覆盖 $25 \mu \mathrm{g} \cdot \mathrm{cm}^{-2}$ 的 CNT 层后, 其厚度显著增加至 $4.0 \mu \mathrm{m}$ 左右(图 $1 \mathrm{~b}$ 和 $1 \mathrm{e}$ ); 与 $\mathrm{Pt} / \mathrm{C}$ 层相比, 添加的 $\mathrm{CNT}$ 层明显更为疏松多孔. 与 CCM-0 相比, 将 CNT 混合添加到催化层中后, 催化层 的整体厚度明显增加, 平均厚度约为 $4.3 \mu \mathrm{m}$ (图 1c 和 1f), 略厚于 CCM-1 催化层, 并且显示出疏松多孔的结构, 孔 隙率和孔径明显高于 CCM-0 和 CCM-1. 为了进一步探 究 CNT 添加至催化层中对催化剂微观结构的影响, 采 用 $\mathrm{N}_{2}$ 吸脱附法对 CCM-0 和 CCM-2 的阴极催化层进行 测试, 结果如图 2 和表 S1 所示. 结果表明, 在催化层中 引入 $\mathrm{CNT}$ 后, 催化层的比表面积和孔体积分别从 CCM-0 的 $30.2 \mathrm{~m}^{2} \cdot \mathrm{g}^{-1}$ 和 $0.07 \mathrm{~cm}^{3} \cdot \mathrm{g}^{-1}$ 增加至 $54.3 \mathrm{~m}^{2} \cdot$ $\mathrm{g}^{-1}$ 和 $0.20 \mathrm{~cm}^{3} \cdot \mathrm{g}^{-1}$, 分别提升了 $79.8 \%$ 和 $185.7 \%$; 加入 CNT 后催化层的初级孔和次级孔的孔径分布以及孔体 积也发生了较为明显的变化. 未加入 CNT 前, 催化层的 初级孔平均孔径为 $2 \sim 3 \mathrm{~nm}$, 次级孔孔径分布在 $8 \sim 70$ $\mathrm{nm}$ 范围, 次级孔孔径峰值为 $25 \mathrm{~nm}$ 左右. 加入 CNT 后, 催化层初级孔平均孔径为 $2 \sim 15 \mathrm{~nm}$, 次级孔孔径分布 在 $15 \sim 90 \mathrm{~nm}$ 范围, 次级孔孔径峰值为 $50 \mathrm{~nm}$ 左右, 而 且初级孔和次级孔的孔容均有所增加. 表明 CNT 的加 入, 在一定程度改变了 $\mathrm{Pt} / \mathrm{C}$ 颗粒的分散状态和聚集状 态, 与 SEM 观测到的结果一致, 这将有利于改善催化层 中反应物和产物水的传输, 从而有利于电池性能的提 升 $^{[23]}$. 


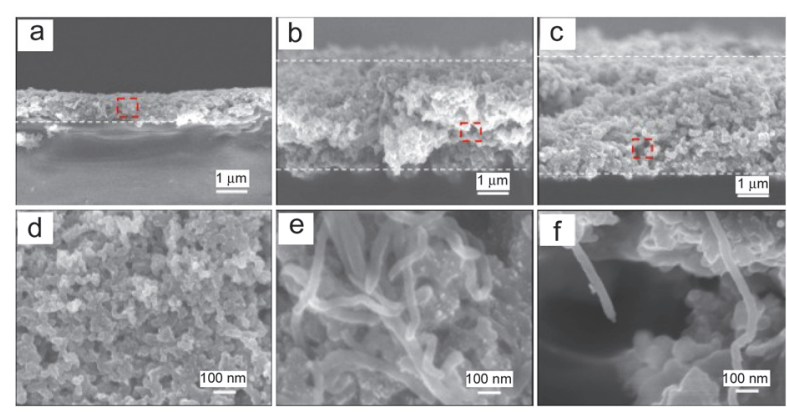

图 1 不同 $\mathrm{CCMs}$ 的阴极催化层断面扫描电镜图

Figure 1 SEM images for cross-section of CCM with different cathode catalytic layer

(a, d) CCM-0, (b, e) CCM-1, and (c, f) CCM-2.
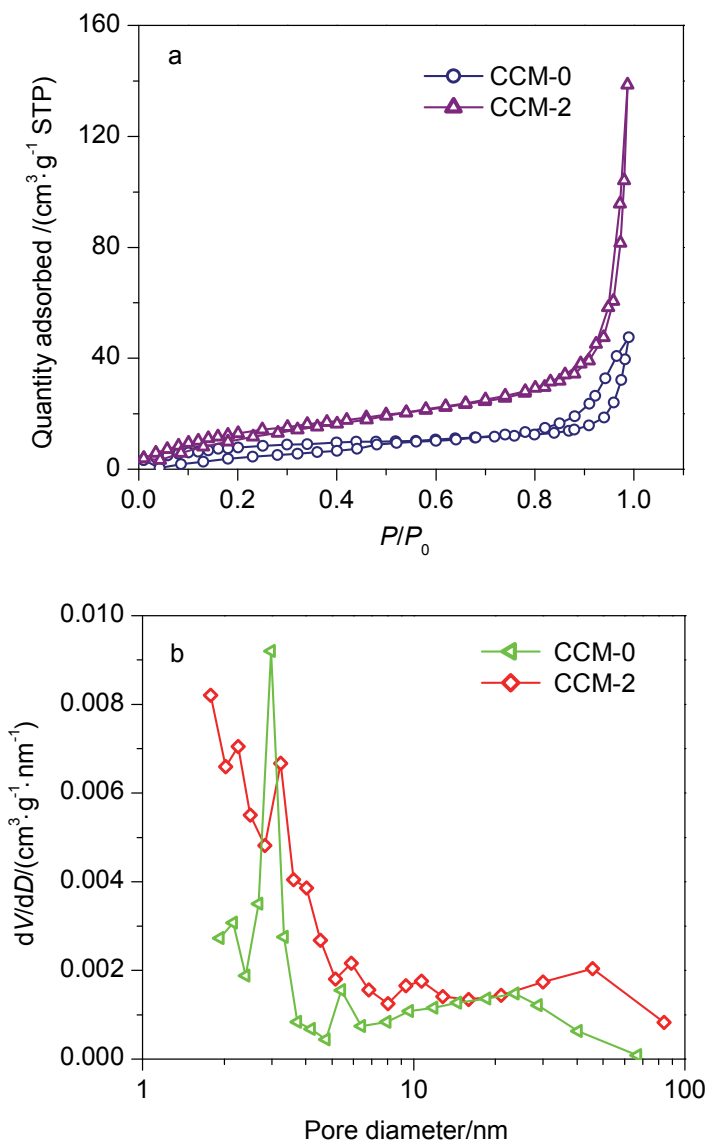

图 $2 \mathrm{CNT}$ 的添加对低铂载量催化层孔径以及孔体积的影响

Figure 2 Effect of CNT addition on pore size and pore volume of low-Pt loading catalytic layer

(a) $\mathrm{N}_{2}$ adsorption isotherm and (b) pore size distribution of CCM-0 and CCM-2 catalytic layer

\subsection{CNT 的添加方式对膜电极性能的影响}

图 3a 比较了 $\mathrm{CNT}$ 的不同添加方式对膜电极输出性 能的影响. 从图中可以看出, 在低铂载量催化层中或表 面添加 CNT 均可明显提升低铂载量膜电极的输出性能, 特别是在大电流密度区, 性能提升更为明显. CCM-1 和 $\mathrm{CCM}-2$ 最高功率密度分别达到 0.639 和 $0.836 \mathrm{~W} \cdot \mathrm{cm}^{-2}$,
较 CCM-0 的 $0.522 \mathrm{~W} \cdot \mathrm{cm}^{-2}$ 分别提升了 $22.4 \%$ 和 $60.0 \%$. 从三种 $\mathrm{CCMs}$ 在 $0.6 \mathrm{~V}$ 工作电压下的交流阻抗结果(图 $3 b$ ) 可以看出, 添加 CNT 后, CCM-1 和 CCM-2 的内阻和 电化学反应电阻均有明显降低. 膜电极内阻从未添加 CNT 时 $(\mathrm{CCM}-0)$ 的 $0.089 \Omega \cdot \mathrm{cm}^{2}$ 降低至表面添加 CNT $(\mathrm{CCM}-1)$ 后的 $0.066 \Omega \cdot \mathrm{cm}^{2}$ 和催化层中均匀添加 $\mathrm{CNT}$ (CCM-2)后的 $0.059 \Omega \cdot \mathrm{cm}^{2}$, 分别下降了 $25.8 \%$ 和 $33.7 \%$; 而且, 添加 CNT 后膜电极的电化学反应电阻从 CCM-0 的 $0.536 \Omega \cdot \mathrm{cm}^{2}$ 分别下降至 CCM-1 的 $0.287 \Omega \cdot \mathrm{cm}^{2}$ 和 $\mathrm{CCM}-2$ 的 $0.252 \Omega \cdot \mathrm{cm}^{2}$, 分别下降了 $46.5 \%$ 和 $53.0 \%$.
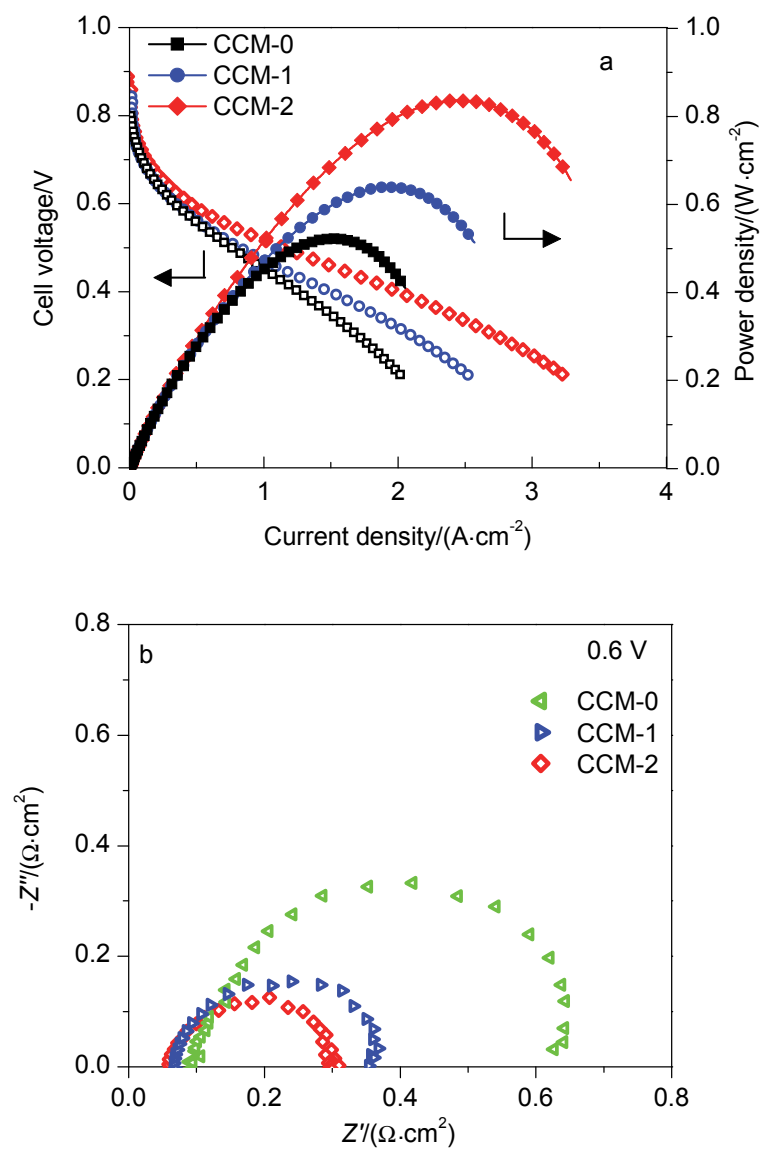

图 3 不同 $\mathrm{CCMs}$ 的电池性能(a)及 $0.6 \mathrm{~V}$ 条件下的交流阻抗谱图(b)(测 试条件: $70{ }^{\circ} \mathrm{C}$, 阴阳极 $100 \%$ 加湿, 无背压)

Figure 3 Cell performance (a) and electrochemical impedance spectra at $0.6 \mathrm{~V}$ (b) of different CCMs (operating condition: $100 \%$ relative humidity, $70{ }^{\circ} \mathrm{C}$ and without back pressure)

上述结果表明仅在低铂载量催化层表面添加 CNT 层后，即可明显降低电池内阻和电化学反应电阻. 对于 低铂载量催化层而言, 由于催化层厚度很薄(如本研究 中为 $1 \mu \mathrm{m})$, 难以保证其与气体扩散层充分接触, 导致 界面接触电阻较大, 在薄层的 $\mathrm{Pt} / \mathrm{C}$ 催化层表面添加一层 $\mathrm{CNT}$ 后, 可以改善催化层 $(\mathrm{CL})$ 与气体扩散层 $(\mathrm{GDL})$ 的接 触, 加快电子传输, 降低界面接触电阻. 此时, 用于电 化学反应的 $\mathrm{Pt} / \mathrm{C}$ 层与 $\mathrm{CCM}-0$ 是相同的, 但其电化学反 应电阻明显降低, 说明 CNT 在 Pt/C 表面的添加改善了 
大电流条件下氧气和水的传输, 减小了浓差极化电阻. 可能的原因为 CNT 层的孔隙率和孔径较大, 且 CNT 层 也较 $\mathrm{Pt} / \mathrm{C}$ 层憎水, 有利于反应物和水的传输.

与在 $\mathrm{Pt} / \mathrm{C}$ 层表面添加 $\mathrm{CNT}$ 层相比, 通过混合方式 将 CNT 添加至 $\mathrm{Pt} / \mathrm{C}$ 层中(CCM-2), 更有利于降低 $\mathrm{CL}$ 与 GDL 的接触电阻和电化学反应电阻. 这主要原因在于 在 $\mathrm{Pt} / \mathrm{C}$ 层中添加 $\mathrm{CNT}$ 后, 由于 $\mathrm{CNT}$ 高的长径比以及优 越的电子导电性能, 提升催化层的整体电子电导率, 同 时还改善了 CL 与 GDL 界面接触, 降低了界面电子传输 电阻; 此外, 将 CNT 添加至催化层中, 催化层的比表面 积和孔径均有所增大, 大幅降低了反应物 $\mathrm{O}_{2}$ 和产物水 的传输阻力, 因此在大电流密度区 $\mathrm{CCM}-2$ 的输出性能 明显提升. 通常, 低 Pt 载量的薄层亲水催化层在大电流 密度条件下容易发生水淹现象 ${ }^{[25,26]}$, 使得 $\mathrm{O}_{2}$ 的传输受 限制, 导致催化层电化学反应阻抗变大. CCM-1 和 CCM-2 电化学反应电阻的下降说明在催化层表面和内 部添加 CNT 均有利于反应产物水的排出, 有利于反应 物氧气传递到催化层中. 而且 CNT 添加至催化层中还 改善了催化层的孔结构和孔体积, 对反应物和产物的传 输与表面添加方式相比更为有利, 因此, 电化学反应阻 抗下降更为明显 ${ }^{[18,23,27]}$.

为了进一步厘清 CNT 在催化层中的作用, 制备了 阴极催化层只含有 $\mathrm{CNT}\left(0.125 \mathrm{mg}_{\mathrm{CNT}}{ }^{\bullet} \mathrm{cm}^{-2}\right)$ 作为催化层 的膜电极(CCM-3), 与其它 $\mathrm{CCM}$ 在相同条件下进行测 试, 其单电池的最高输出功率只有 $2.9 \mathrm{~mW} \cdot \mathrm{cm}^{-2}$, 而 0.6 $\mathrm{V}$ 工作电压下电极反应电阻高达 $1002.7 \Omega \cdot \mathrm{cm}^{2}$ (参见支 持信息图 S1 和表 $\mathrm{S} 2$ ), 表明 $\mathrm{CCM}-2$ 性能的提升并非 $\mathrm{CNT}$ 的催化作用, 而主要是由于 CNT 的加入改变了催 化层微观结构, 进而改善了催化层中氧气和水的传输以 及降低了催化层和气体扩散层的界面接触电阻.

为了探究两种 CNT 添加方式对 CCM 性能提升时内 阻降低获益、传输加快获益的权重, 本工作结合 EIS 结 果对 $I-V$ 曲线进行了 IR 降校正, 结果如图 4 和表 1 所示. 图 4 展示了 $\mathrm{CNT}$ 添加方式对电池内阻及传质电阻引起 的电压降对电池极化曲线的影响. 在电流密度 $2 \mathrm{~A} \cdot \mathrm{cm}^{-2}$ 下，与 CCM-0 相比, CCM-1 的欧姆压降下降了 $46 \mathrm{mV}$, 由传质引起的电压降降低了 $57 \mathrm{mV}$, 欧姆压降和传质电 压降下降对电池性能提升的贡献率分别为 $44.7 \%$ 和
$55.3 \%$. 以上结果说明在低铂载量催化层表面添加一层 CNT 层即可同时改善电池内阻和气体传输. 同样条件 下, CCM-2 的欧姆压降与 CCM-0 相比下降了 $60 \mathrm{mV}$, 由 传质加快引起的电压降下降了 $124 \mathrm{mV}$, 说明改善传质 是电池输出性能提升的主要因素(权重为 $67.4 \%$ ). 而且, 与 CCM-1 相比, CCM-2 输出功率的提升幅度主要是由 于传质改善获得增益更大，即与表面添加方式相比，混 合添加方式的传质效率提高了 $21.9 \%$. 原因是在 $\mathrm{Pt} / \mathrm{C}$ 层 中添加 CNT 使催化层变得均匀且疏松多孔, 更有利于 反应物氧气的传输和产物水的排出.
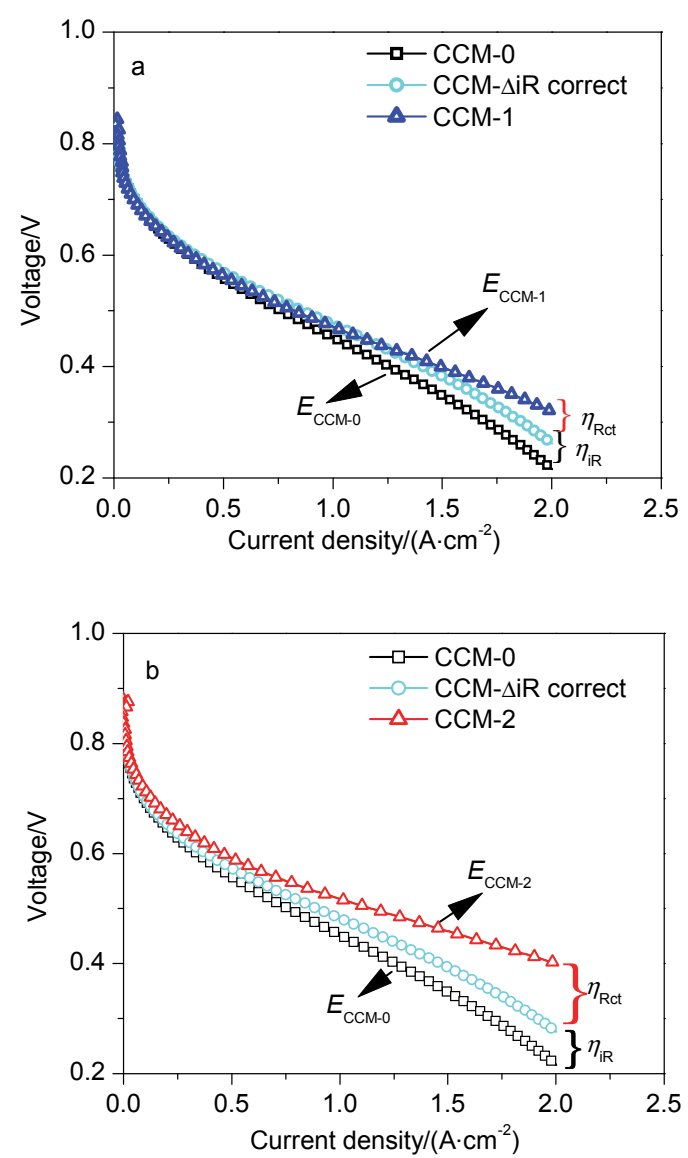

图 $4 \mathrm{CNT}$ 的添加方式对不同 $\mathrm{CCM}$ 输出性能提升的分析

Figure 4 Analysis for the adding mode of CNT to improve cell performance

(a) $\mathrm{CCM}-1$, (b) $\mathrm{CCM}-2$

表 1 不同 CCMs 的电池性能、交流阻抗以及 CNT 添加对 CCM 的电池性能影响原因分析信息表 ${ }^{a}$

Table 1 Information of the cell performance, EIS and performance gain from internal resistance reduction and mass transmission enhancement of different CCMs

\begin{tabular}{ccccccccc}
\hline Sample & $P_{\max } /\left(\mathrm{W} \cdot \mathrm{cm}^{-2}\right)$ & $P_{0.6 \mathrm{~V}} /\left(\mathrm{W} \cdot \mathrm{cm}^{-2}\right)$ & $\begin{array}{c}R_{\mathrm{s}} /\left(\Omega \cdot \mathrm{cm}^{2}\right) \\
(@ 0.6 \mathrm{~V})\end{array}$ & $\begin{array}{c}R_{\mathrm{ct}} /\left(\Omega \cdot \mathrm{cm}^{2}\right) \\
(@ 0.6 \mathrm{~V})\end{array}$ & $\begin{array}{c}\eta_{\mathrm{iR}} / \mathrm{mV} \\
\left(@ 2 \mathrm{~A} \cdot \mathrm{cm}^{-2}\right)\end{array}$ & $\begin{array}{c}\eta_{\mathrm{Rcc}} / \mathrm{mV} \\
\left(@ 2 \mathrm{~A} \cdot \mathrm{cm}^{-2}\right)\end{array}$ & $\begin{array}{c}\text { 欧姆电压降 } \\
\text { 获益权重 } / \%\end{array}$ & $\begin{array}{c}\text { 传质电压降 } \\
\text { 获益权重 } / \%\end{array}$ \\
\hline $\mathrm{CCM}-0$ & 0.522 & 0.202 & 0.089 & 0.536 & & & & \\
$\mathrm{CCM}-1$ & 0.639 & 0.210 & 0.066 & 0.287 & 46 & 57 & 44.7 & 55.3 \\
$\mathrm{CCM}-2$ & 0.836 & 0.273 & 0.059 & 0.251 & 60 & 124 & 32.6 & 67.4 \\
\hline
\end{tabular}

${ }^{a} P_{\max }$ 和 $P_{0.6 \mathrm{~V}}$ 分别是 $\mathrm{CCMs}$ 的最大功率密度和 $0.6 \mathrm{~V}$ 时的功率密度; $R_{\mathrm{s}}$ 为膜内阻, $R_{\mathrm{ct}}$ 为电化学反应电阻; $\eta_{\mathrm{iR}}$ 为 $\mathrm{CCM}-1$ 和 CCM- 2 相较于 CCM- 0 的内阻校正 值, $\eta_{\mathrm{Rct}}$ 为 CCM-1 和 CCM-2 相较于 CCM-0 由于质量传输增强而减少的过电势. 
为了考察 $\mathrm{CNT}$ 的添加对 $\mathrm{Pt}$ 催化剂的利用率是否有 影响, 对 CCM-0, CCM-1, CCM-2 阴极催化层进行了循 环伏安测试, 结果如图 5 所示. 从图 $5 \mathrm{a}$ 的 $\mathrm{CV}$ 曲线看出, 添加 CNT 后 CCM-1 的氢区面积和 CCM-0 无明显差异, 而 CCM-2 氢区峰面积明显大于 CCM- 0 和 CCM-1. 对图 $5 \mathrm{a}$ 中 $\mathrm{CV}$ 曲线的氢区进行积分, 利用 Eq. 1(见支持信息) 计算 $\mathrm{Pt}$ 催化剂的电化学活性面积 $(\mathrm{ECSA})^{[27 \sim 30]}$, 结果如 图 $5 \mathrm{~b}$ 所示. 从图 $5 \mathrm{~b}$ 可以看出, 三者的 ECSA 变化趋势 和电池性能变化趋势一致. CCM-0 阴极催化层的 ECSA 为 $28.9 \mathrm{~m}^{2} \cdot \mathrm{g}^{-1}$, 而 CCM-1 和 CCM-2 的 ECSA 分别达到 $29.7 \mathrm{~m}^{2} \cdot \mathrm{g}^{-1}$ 和 $37.3 \mathrm{~m}^{2} \cdot \mathrm{g}^{-1}$, 较 CCM-0 分别提升了 $2.8 \%$ 和 $29.1 \%$. 通过透射电子显微镜(TEM) 对 Pt/C 纳米颗粒 表征(图 5c), 并对其粒径进行了统计(图 5d), 得知 $\mathrm{Pt}$ 的 平均粒径 $D=3.3 \pm 0.08 \mathrm{~nm}$. 通过 Eq. 2(见支持信息)计 算得到 CCM- 0, CCM-1, CCM-2 的 Pt 利用率分别为 $34.4 \%, 35.6 \%, 44.7 \%$ (图 5b). 以上结果说明 CNT 添加至 催化层中, 改善了催化层的三相界面, 从而显著提升了 Pt 催化剂的利用效率. 可能的原因是混合方式添加 $\mathrm{CNT}$ 时, 在墨水的配制过程中 CNT 改善了 $\mathrm{Pt} / \mathrm{C}$ 的分散 性, 减缓了 $\mathrm{Pt} / \mathrm{C}$ 的颗粒的团聚现象, 使更多的 $\mathrm{Pt}$ 纳米颗 粒可以与聚合物电解质接触, 从而增大了 $\mathrm{Pt}$ 的电化学
面积和利用效率 ${ }^{[23,31]}$.

\subsection{CNT 的添加量对膜电极的影响}

在确定阴极催化层中 CNT 较优的添加方式后, 进 一步研究了 CNT 的在催化层中的添加量对电池性能的 影响, 结果如图 6 所示. 在本文测试的 CNT 添加量范围 内, 膜电极的输出性能均有提升; 且随着 CNT 添加量的 增加, 膜电极的最大输出功率密度以及 $0.6 \mathrm{~V}$ 工作电压 下的输出功率密度均呈现先增大后减小的趋势(图 6b). 当 $\mathrm{CNT}$ 的添加量为 $37.5 \mu \mathrm{g} \cdot \mathrm{cm}^{-2}$ 时, 膜电极的输出性能 最优, 其最高输出功率密度达到 $0.91 \mathrm{~W} \cdot \mathrm{cm}^{-2}$, 较 $\mathrm{CCM}-0$ 提升了 $74.3 \%$. 图 $6 \mathrm{c}$ 为不同 CNT 添加量的 $\mathrm{CCMs}$ 在 $0.6 \mathrm{~V}$ 工作电压下的 EIS 图谱. 结果表明 CNT 添加量从 0 增加至 $37.5 \mu \mathrm{g} \cdot \mathrm{cm}^{-2}$, 膜电极的内阻由 0.089 $\Omega \cdot \mathrm{cm}^{2}$ 连续下降至 $0.047 \Omega \cdot \mathrm{cm}^{2}$, 同时电荷转移电阻由 $0.536 \Omega \cdot \mathrm{cm}^{2}$ 降低至 $0.194 \Omega \cdot \mathrm{cm}^{2}$ (如图 6d 所示). 其主要 原因是提高在低 $\mathrm{Pt}$ 载量的薄层亲水电极催化层中 $\mathrm{CNT}$ 添加量增加了催化层的厚度, 改善了 CL 和 GDL 的接 触，降低了界面电阻; 而且由于 CNT 的高的长径比和优 越电子导电性，改善了催化层的电子电导. 另外，不同 含量 CNT 的添加使催化层的孔隙率和孔径增大，显著
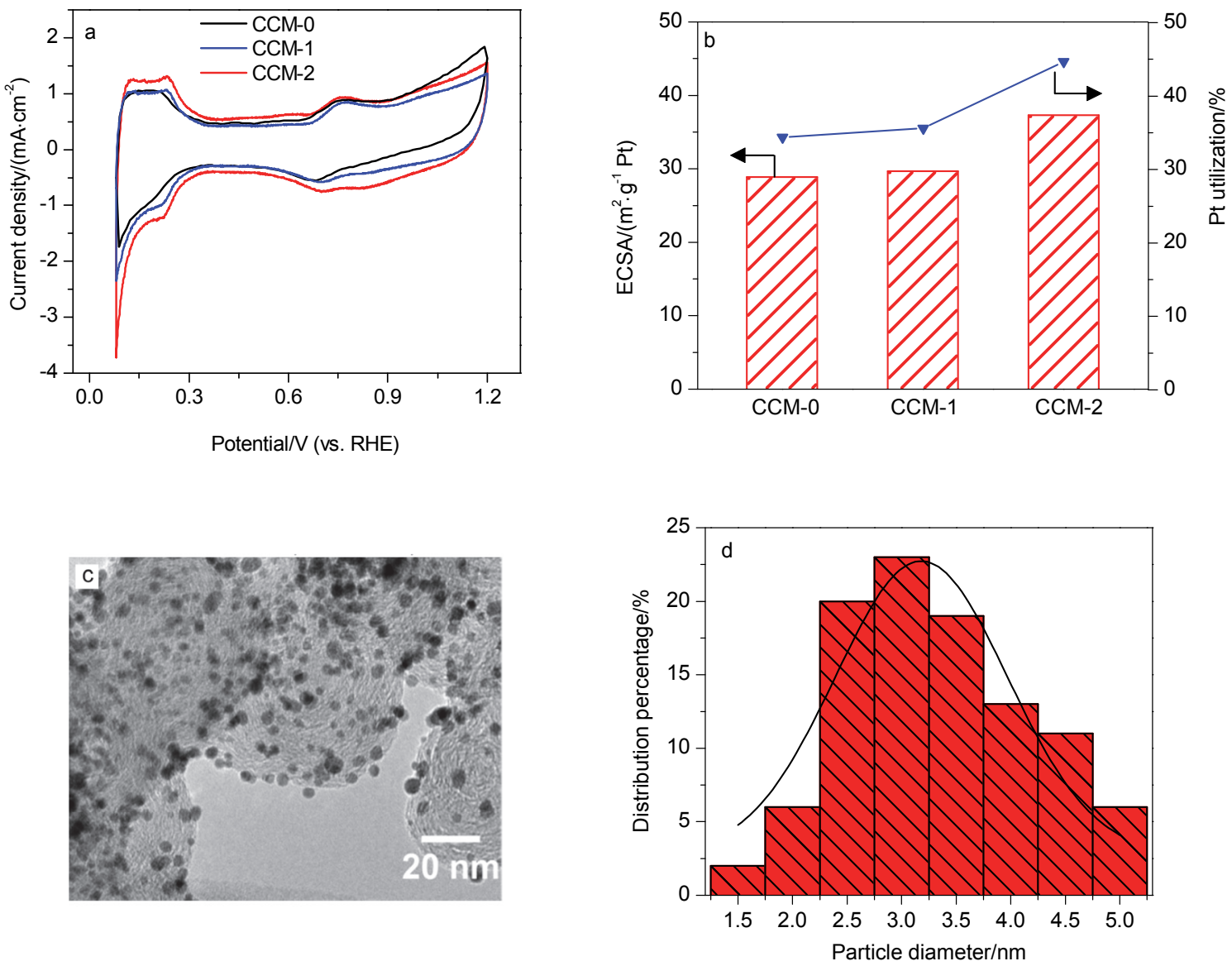

图 5 (a) 三种 CCMs 的阴极催化层的循环伏安曲线、(b) 电化学活性面积和铂的利用率、(c) $\mathrm{Pt} / \mathrm{C}$ 催化剂的透射电镜图和(d) 铂颗粒的粒径分布. Figure 5 Cyclic voltammograms curves (a), electrochemical surface and Pt utilization of three different CCMs (b), TEM image of Pt/C catalyst (c) and size distribution of Pt particles (d) 

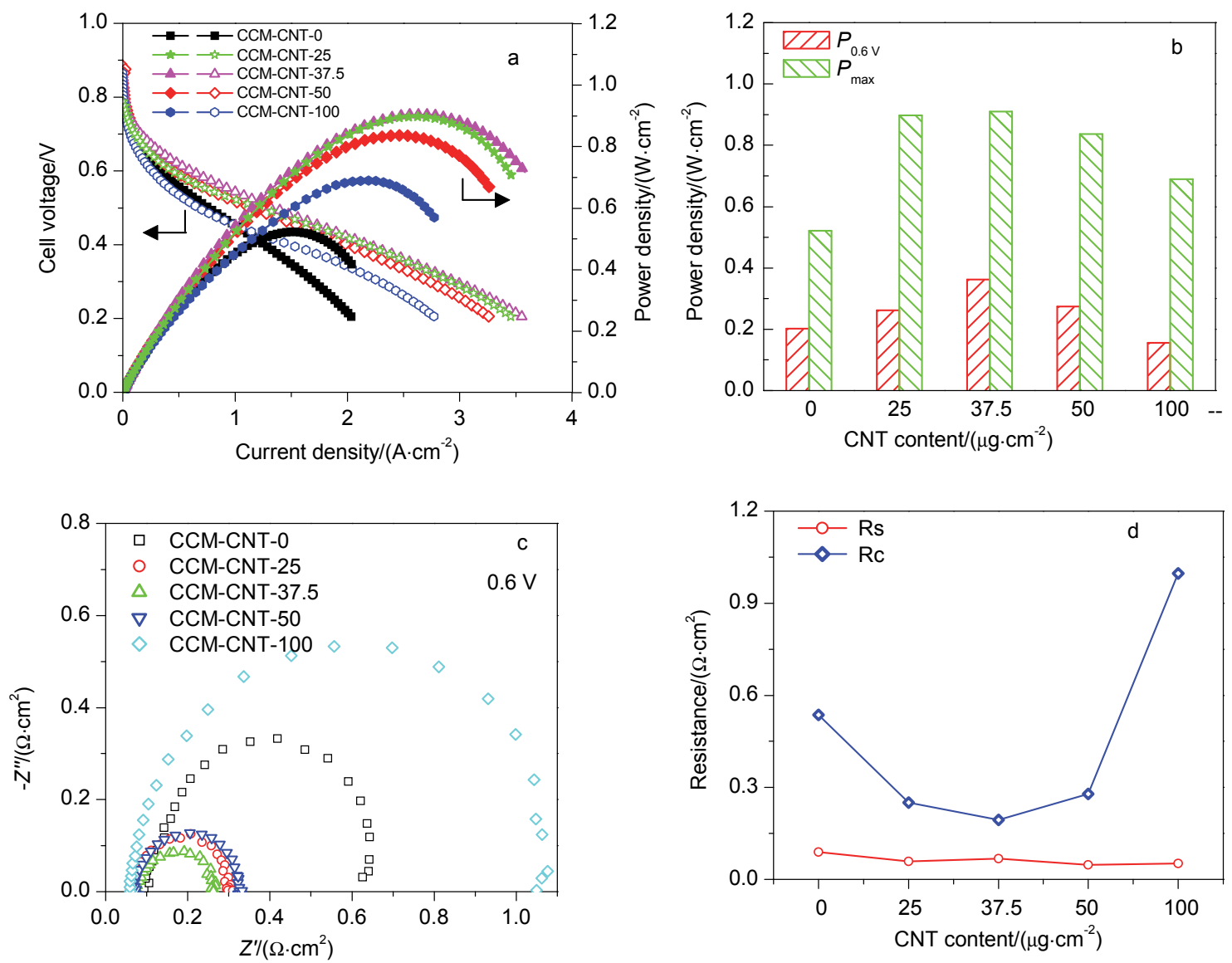

图 6 (a, b)不同 CNT 量的 CCMs 的电池性能和(c, d) $0.6 \mathrm{~V}$ 条件下的交流阻抗谱图(测试条件: $70^{\circ} \mathrm{C}$, 阴阳极 100\%加湿, 无背压)

Figure 6 (a, b) Cell performance of the CCMs with different content of CNT and (c, d) electrochemical impedance spectroscopy at $0.6 \mathrm{~V}$ of the CCMs with different content of CNT (test conditions: $70{ }^{\circ} \mathrm{C}, 100 \%$ relative humidity for both anode and cathode, without back pressure)

降低了 $\mathrm{O}_{2}$ 和水的传输阻力, 例如在大电流密度 $2 \mathrm{~A} \cdot$ $\mathrm{cm}^{-2}$ 下, 输出电压由未添加 CNT (CCM-0)的 $0.217 \mathrm{~V}$ 提 高至 CNT 最优添加量 $37.5 \mu \mathrm{g} \cdot \mathrm{cm}^{-2}$ 时的 $0.421 \mathrm{~V}$, 由传 输引起的浓差极化现象明显减小. 该结果与文献报道一 致 ${ }^{[26]}$. 继续将阴极催化层中 $\mathrm{CNT}$ 的添加量由 37.5 $\mu \mathrm{g} \bullet \mathrm{cm}^{-2}$ 增加至 $100 \mu \mathrm{g} \cdot \mathrm{cm}^{-2}$ 时, 膜电极的内阻由 0.047 $\Omega \cdot \mathrm{cm}^{2}$ 反而增大至 $0.052 \Omega \cdot \mathrm{cm}^{2}$, 且电化学反应电阻也由 最低值 $0.194 \Omega \cdot \mathrm{cm}^{2}$ 增大至 $0.997 \Omega \cdot \mathrm{cm}^{2}$ (图 6c $\sim 6 \mathrm{~d}$ ). 出 现这一变化趋势可能是因为过高的 CNT 添加量导致催 化层厚度过大, 使电子、质子、气体传输路径变长, 增 加了传质阻力, 导致产物水无法顺利排出, 氧气传输受 阻 ${ }^{[32]}$; 在高电流密度区 $2 \mathrm{~A} \cdot \mathrm{cm}^{-2}$ (图 6a) 时, 放电电压也 由 CNT 添加量为 $37.5 \mu \mathrm{g} \cdot \mathrm{cm}^{-2}$ 时的 $0.421 \mathrm{~V}$ 降低至 CNT 载量为 $100 \mu \mathrm{g} \cdot \mathrm{cm}^{-2}$ 时的 $0.342 \mathrm{~V}$, 与文献报道规律类 似 ${ }^{[33]}$.

\section{3 结论}

探究了阴极催化层中 CNT 的添加方式和添加量对 低铂膜电极电池性能的影响, 并从微观形貌层面探究了 其构效关系. 结果表明, 在催化层中混合添加 CNT 的方
式有利于优化其孔隙结构, 从而降低接触电阻和传质电 阻, 同时有利于提高催化层中铂的利用率. 混合添加 $\mathrm{CNT}$ 的 CCM 最大输出功率较未添加和分步添加 CCM 的最高输出功率分别提升了 $60.0 \%$ 和 $30.8 \%$. 另外, 通 过对混合添加的方式中 CNT 的添加量的进一步优化, $\mathrm{CNT}$ 的最优添加量为 $37.5 \mu \mathrm{g} \cdot \mathrm{cm}^{-2}$ 时, 电池的最大输出 功率达到 $0.91 \mathrm{~W} \cdot \mathrm{cm}^{-2}\left(70{ }^{\circ} \mathrm{C}\right.$ 、阴阳极 $100 \%$ 加湿和无背 压条件). 总之, 本研究通过在低 $\mathrm{Pt}$ 载量的质子交换膜 燃料电池的催化层中引入 CNT 的方式, 从微观结构改 善了催化层和气体扩散层的接触界面, 加速了传质过 程, 从而大幅度提高了电池性能, 对催化层微观形貌与 质子交换膜燃料电池性能之间的构效关系的理解有一 定意义.

\section{4 实验部分}

本研究中膜电极的有效面积为 $2 \mathrm{~cm} \times 2 \mathrm{~cm}$. 阳极 催化层的制备方法为: 按照一定比例将 $\mathrm{Pt} / \mathrm{C}$ 催化剂、异 丙醇与 $5 \mathrm{wt} \% \mathrm{Nafion}^{\circledR}$ 溶液混合均匀得到催化剂墨水, 然后采用超声喷涂方法将在 Nafion 211 膜上得到阳极催 化层, 其中铂载量为 $0.1 \mathrm{mg} \bullet \mathrm{cm}^{-2}$, 催化层中 Nafion 的 
含量为 $20 \mathrm{wt} \%$.

阴极催化层的制备：按照一定比例将 $\mathrm{Pt} / \mathrm{C}$ 催化剂、 异丙醇与 $5 \mathrm{wt} \% \mathrm{Nafion}^{\circledR}$ 溶液混合均匀制备催化剂墨水, 然后在上述含有阳极催化层的 Nafion 211 膜的另一侧上 采用超声喷涂方法得到阴极催化层, 记为 CCM-0; 若在 上述阴极催化层上进一步喷涂由 CNT、异丙醇和 Nafion ${ }^{\circledR}$ 溶液组成的墨水, 形成双层结构的催化层, 记为 CCM-1; 将一定比例的 CNT、Pt/C 催化剂、异丙醇与 5 $w t \%$ Nafion ${ }^{\circledR}$ 溶液超声混合均匀后喷涂在上述含有阳极 催化层的 Nafion 211 膜的另一侧上得到复合阴极催化 层, 记为 CCM-2. 三种阴极催化层中 $\mathrm{Pt}$ 载量均为 0.1 $\mathrm{mg} \cdot \mathrm{cm}^{-2}$; 此外, CCM-1 和 CCM-2 中 CNT 的含量为 25 $\mu \mathrm{g} \bullet \mathrm{cm}^{-2}$.

将一定比例的 CNT、异丙醇与 $5 \mathrm{wt} \% \mathrm{Nafion}^{\circledR}$ 溶液 超声混合均匀后喷涂在上述含有阳极催化层的 Nafion 211 膜的另一侧上得到 CNT 的阴极催化层(无 $\mathrm{Pt} / \mathrm{C}$ 催化 剂), 记为 CCM-3, 其中 CNT 的含量为 $0.125 \mathrm{mg} \bullet \mathrm{cm}^{-2}$. 催化层的微观结构表征用扫描电子显微镜、透射电 子显微镜和氮物理吸附法, 具体见支持信息. 燃料电池 单电池性能测试采用 Greenlight G20 燃料电池测试系统 和普林斯顿电化学工作站(Princeton PARSTAT 4000+) 进行. 测试条件为 $70{ }^{\circ} \mathrm{C} 、 100 \%$ 加湿和无背压, $I-V$ 极化 曲线扫速为 $10 \mathrm{mV} \cdot \mathrm{s}^{-1}$; 膜电极阻抗采用恒压工作模式 $(0.6 \mathrm{~V})$, 阻抗频率范围为 $100 \mathrm{kHz} \sim 0.1 \mathrm{~Hz}$, 偏压为 10 $\mathrm{mV} ; \mathrm{Pt}$ 催化剂的 ECSA 采用循环伏安法, 电压范围为 $0.08 \sim 1.2 \mathrm{~V}$, 扫速为 $50 \mathrm{mV} \cdot \mathrm{s}^{-1}$, 其中 $\mathrm{Pt}$ 的电化学活性

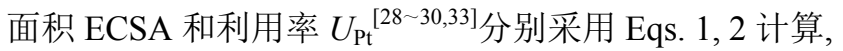
具体见支持信息.

\section{References}

[1] Chu, S.; Majumdar, A. Nature 2012, 488, 294.

[2] Wang, J.; Wang, H.; Fan, Y. Engineering 2018, 4, 352.

[3] Li, H.; Li, L.; Chen, S.; Zhang, Y.; Li, G. Chin. J. Chem. 2017, 35, 903.

[4] He, X.; Gang, M.; He, G.; Yin, Y.; Cao, L.; Wu, H.; Jiang, Z. Chin. J. Chem. 2017, 35, 673

[5] Hou, I.; Shetti, V.; Huang, S.; Liu, K.; Chao, C.; Lin, S.; Lin, Y.; Chen, L; Luh, T. Org. Chem. Front. 2017, 4, 773.
[6] Proietti, E.; Jaouen, F.; Lefevre, M.; Larouche, N.; Tian, J.; Herranz, J.; Dodelet, P. Nat. Commun. 2011, 2, 416.

[7] Lee, H.; Park, J.; Kim, D.; Lee, T. J. Power Sources 2004, 131, 200.

[8] Peng, S.; Xu. X.; Zhang, J.; Liu, Y.; Lu, S.; Xiang, Y. Acta Chim. Sinica 2015, 73, 137. (彭思㑆, 徐金佛, 劲, 刘神阳, 卢善富, 相 艳, 化学学报, 2015, 73, 137.)

[9] Shin, S.; Kim, A.; Um, S. Int. J. Hydrogen Energy 2016, 41, 9507.

[10] Huang, T.; Shen, H.; Jao, T.; Weng, F.; Su, A. Int. J. Hydrogen Energy 2012, 37, 13872.

[11] Zhu, C.; Hai, Y.; Zhao, Z.; Yang, Y. Acta Chim. Sinica 2018, 76, 30. (朱婵, 海洋, 赵志刚, 阳耀月, 化学学报, 2018, 76, 30.)

[12] Zhong, G.; Wang, H.; Yu, H.; Peng, F. Acta Chim. Sinica 2017, 75, 943. (钟国玉, 王红娟, 余皓, 彭峰, 化学学报, 2017, 75, 943.)

[13] Wu, Y.; Liao, S. Chin. J. Power Source 2012, 36, 1755. (吴燕妮, 廖 世军，电源技术, 2012, 36, 1755.)

[14] Chen, X.; Yan, H.; Xia, D. Acta Chim. Sinica 2017, 75, 189. (陈金金, 鄢慧君, 夏定国, 化学学报, 2017, 75, 189.)

[15] Yuan, P.; Chen, J.; Pan, D.; Bao, X. Acta Chim. Sinica 2016, 74, 603. (袁佩, 陈建, 潘登, 鲍晓军, 化学学报, 2016, 74, 603.)

[16] Chen, G.; Zhao, B.; Wang, C.; Jing, P.; Xiao, Y.; Niu, M.; Zhao, P. Chem. Bull. 2016, 79, 9. (陈光颖, 赵波, 王诚, 荆平，肖宇，牛萌， 赵鹏程, 化学通报, 2016, 79, 9.)

[17] Li, L.; Jiang, D.; Zeng, R.; Wang, S.; Jiang, L. Rare Metals 2017, 41, 648. (李琳, 姜东, 曾蓉, 王树茂, 蒋利军, 稀有金属, 2017, 41, 648.)

[18] Ishikawa, H.; Sugawara, Y.; Inoue, G.; Kawase, M. J. Power Sources 2018, 374, 196.

[19] Shin, S.; Kim, A.; Um, S. Electrochim. Acta 2016, 207, 187.

[20] Cho, J.; Kim, J.; Prabhuram, J.; Hwang, S.; Ahn, D.; Ha, H.; Kim, S. J. Power Sources 2009, 187, 378.

[21] Pollet, B. G.; Goh, J. T. E. Electrochim. Acta 2014, 128, 292.

[22] Tian, Z.; Lim, S.; Poh, C.; Tang, Z.; Xia, Z.; Luo, Z.; Shen, P.; Chua, D.; Feng, Y.; Shen, Z.; Lin, J. Adv. Energy Mater. 2011, 1, 1205.

[23] Hou, S.; Chi, B.; Liu, G.; Ren, J.; Song, H.; Liao, S. Electrochim. Acta 2017, 253, 142.

[24] Suzuki, T.; Hashizume, R.; Hayase, M. J. Power Sources 2015, 286, 109.

[25] Ji, M.; Wei, Z. Energies 2009, 2, 1057.

[26] Zenyuk, I.; Das, P.; Weber, A. J. Electrochem. Soc. 2016, 163, F691.

[27] Lee, M.; Uchida, M.; Tryk, D.; Uchida, H.; Watanabe, M. Electrochim. Acta 2011, 56, 4783.

[28] Koh, J.; Jeon, Y.; Cho, Y.; Kim, J.; Shul, Y. J. Mater. Chem. A 2014, 2, 8652 .

[29] Park, Y.; Tokiwa, H.; Kakinuma, K.; Watanabe, M.; Uchida, M. J. Power Sources 2016, 315, 179.

[30] Uchida, M.; Park, Y.; Kakinuma, K.; Yano, H.; Tryk, D.; Kamino, T.; Uchida, H.; Watanabe, M. Phys. Chem. Chem. Phys. 2013, 15, 11236.

[31] Oh, E.; Hempelmann, R.; Nica, V.; Radev, I.; Natter, H. J. Power Sources 2017, 341, 240.

[32] Xu, X.; Peng, S.; Zhang, J.; Lu, S.; Xiang, Y. Acta Chim. Sinica 2016, 74, 271. (徐金鈼思㑆, 张劲, 卢善富, 相艳, 化学学报, 2016, 74, 271.)

[33] Jeon, Y.; Kim, D.; Koh, J.; Ji, Y.; Kim, J.; Shul, Y. Sci. Rep. 2015, 5, 16394.

(Zhao, C.) 